\title{
Avaliação do Uso do Tecimento sobre o Nível de Diluição e Geometria do Cordão de Solda na Soldagem TIG com Alimentação Automática de Arame Frio
}

\author{
Edvan Cordeiro de Miranda ${ }^{1}$, Cleiton Carvalho Silva ${ }^{1}$, Marcelo Ferreira Motta ${ }^{1}$, Hélio Cordeiro de Miranda ${ }^{1}$, \\ Jesualdo Pereira Farias ${ }^{1}$ \\ 1 Laboratório de Engenharia de Soldagem, Departamento de Engenharia Metalúrgica e de Materiais, Universidade Federal do \\ Ceará, Fortaleza, CE, Brasil.
}

Recebido: 14 Fev., 2015

Aceito: 12 Jun., 2015

E-mail: edvmiranda@yahoo.com.br (ECM)
Resumo: Este trabalho tem por objetivo estudar a influência do tecimento na soldagem TIG com alimentação de arame frio em único passe visando à aplicação futura em revestimento de ligas de níquel, considerando a análise das características geométricas e diluição, de forma a adequar estes revestimentos para as indústrias do Setor de Petróleo e Gás Natural. A baixa diluição é necessária para evitar a redução das propriedades de resistência à corrosão dos revestimentos. As soldagens foram realizadas utilizando um robô industrial e um alimentador automático de arame frio. A liga usada como metal de adição foi a AWS ER NiCrMo-3 (Inconel 625) e o substrato foi o aço ASTM A516 Gr. 60. Foram realizadas soldagens com alimentação automática de arame frio na condição sem tecimento e com tecimento tipo triangular, variando a energia em três níveis. Os resultados mostraram que o uso do tecimento influencia consideravelmente a geometria do cordão de solda e o nível de diluição. Com uso do tecimento o reforço do cordão de solda reduziu e a largura aumentou, proporcionando uma melhor distribuição de material sobre a peça, reduzindo com isso a razão reforço/largura (R/L), parâmetro este que indica o grau de convexidade do cordão de solda. Uma razão R/L alta, indica que o grau de convexidade pode ser excessivo, dificultando com isso uma adequada sobreposição de passes para soldagem posterior de revestimentos, com a possibilidade de formação de vazios entre os mesmos. Quanto a penetração e a diluição, ambas apresentaram comportamentos similares, com redução de forma significativa nas condições em que o tecimento é empregado. Estes resultados mostram que o uso do tecimento garante uma combinação de baixa razão R/L com baixa diluição, condição esta desejável para soldagem de revestimentos.

Palavras-chave: Soldagem TIG; Tecimento; Diluição; Ligas de níquel.

\section{Wave Arc Movement Influence on the Dilution Level and on Weld Bead Geometry Using TIG Welding Process with Automatic Cold Wire Feeding}

\begin{abstract}
This work aims to study the influence of arc waving in GTAW process with cold wire feeding deposited in single pass in order to use nickel-based alloys as coating to of petroleum and natural gas industries, where low dilution is needed to avoid reduction in corrosion resistance of the coatings. The welds were made using an robot workbench and a cold wire feeder system. The alloy used as filler metal was AWS ER NiCrMo-3 (Inconel 625) and the substrate was ASTM A516 Gr 60 steel plates. Welds were carried out with automatic cold wire feeding varying without arc waving and with triangular type arc waving. In addition the heat input was changed at three levels. The results showed that the use of arc waving has considerably influence on the geometry of the weld bead and the level of dilution. With use of arc waving the weld bead reinforcement was reduced and the width were increased, providing a better distribution of material on the workpiece surface, thereby reducing the reinforcement/width (R/W) ratio. This parameter indicates the degree of convexity of the weld bead. A high R/W ratio indicates that the degree of convexity may be excessive; thereby hampering an adequate overlap between the passes aiming to produce weld overlay without voids between them. Penetration and dilution showed similar behaviors, with significantly reduction under conditions where the arc waving was applied. These results show that the use of arc waving ensures a combination of low R/W ratio with low dilution, condition that is desirable to weld coatings.
\end{abstract}

Key-words: GTAW; Arc waving; Dilution; Nickel Alloy. 
Avaliação do Uso do Tecimento sobre o Nível de Diluição e Geometria do Cordão de Solda na Soldagem TIG com Alimentação Automática de Arame Frio

\section{Introdução}

A degradação de equipamentos nas indústrias do Setor de Petróleo e Gás Natural devido a corrosão vêm se intensificando cada vez mais devido a fatores como alterações na corrosividade dos petróleos e alterações nas condições de processamento [1-3]. Uma solução para minimizar a degradação dos equipamentos, seria fabricar equipamentos que combinem ótimas propriedades mecânicas com excelente resistência à corrosão, principalmente em altas temperaturas. Alguns materiais apresentam estas características como os aços inoxidáveis ferríticos, aços inoxidáveis austeníticos, aços inoxidáveis martensíticos, aços inoxidáveis duplex, aços inoxidáveis endurecidos por precipitação e as ligas de níquel [4-7]. Atualmente, os materiais mais utilizados são os aços inoxidáveis devido ao seu menor custo quando comparado com as ligas de níquel, todavia, as ligas de níquel apresentam uma boa alternativa devido a sua excelente resistência à corrosão sob-tensão e, por isso, sua aplicação em equipamentos utilizados na indústria do petróleo e gás natural tem crescido.

Dentre as Ligas de níquel, têm-se a INCONEL 625 que apresenta boa combinação entre resistência à tração, à fluência, fácil fabricação, boa soldabilidade e boa resistência à corrosão em baixa e altas temperatura, alta resistência à corrosão em meio aquoso contendo cloretos quando comparada com aços inoxidáveis, boa resistência à corrosão sob tensão e, em geral, boa performance em outros tipos de ambientes agressivos [8]. Esta liga apresenta um alto teor de $\mathrm{Cr}$, elemento responsável pelo aumento da resistência à corrosão e oxidação, mediante a formação de uma camada passivadora do óxido de cromo $\left(\mathrm{Cr}_{2} \mathrm{O}_{3}\right)$. Além disso, possui a adição de molibdênio que contribui significativamente para o aumento da resistência à corrosão, especialmente corrosão localizada (pites e frestas) [9].

A fabricação de equipamentos empregando integralmente estas ligas pode ser considerada inviável devido ao seu elevado custo. Uma alternativa interessante pode ser a fabricação de equipamentos e tubulações com materiais menos nobres como aços carbono e aços baixa liga e, em seguida, a aplicação de revestimentos com ligas que apresentem uma combinação de excelentes propriedades mecânicas e alta resistência à corrosão para resistirem à agressividade causada pelo meio corrosivo [10].

Um dos processos que pode ser empregado para soldagem de revestimento com ligas de níquel é o processo tungsten inert gas (TIG) com alimentação de arame frio. Este processo, além de garantir alta qualidade do depósito devido a alta estabilidade do arco, apresenta um bom controle de energia. Devido a alimentação de arame ser independente do arco, a quantidade de calor que chega a peça é reduzida, podendo acarretar em menores níveis de diluição [11]. Estas características do TIG com alimentação de arame frio viabilizam sua aplicação para revestimento de componentes ou peças das indústrias do Setor de Petróleo e Gás Natural.

O controle da diluição é importante na soldagem de metais dissimilares, bem como na deposição de revestimentos especiais sobre um substrato de composição química distinta. Valores baixos de diluição são desejáveis para manter a composição química do metal de adição e, conseqüentemente sua resistência a corrosão [11-13]. Revestimentos com diluição superior a $10 \%$ aumentam o teor de ferro do metal de solda e reduzem consideravelmente a resistência à corrosão das ligas de níquel. Baseado nisso, o teor de ferro permitido nos revestimentos destinados a condições severas de serviço deve ser igual ou inferior a 5\%p. de sua composição química global [14].

Embora existam trabalhos na literatura sobre a exploração dos parâmetros de soldagem visando a deposição de revestimentos [15-20], poucos dedicam-se a avaliar o efeito do tecimento [21]. Baseado nesta carência este trabalho tem como objetivo estudar a influência do tecimento na soldagem TIG com alimentação de arame frio em único passe visando à aplicação futura em revestimento de ligas de níquel, considerando a análise das características geométricas e diluição, de forma a adequar estes revestimentos para as indústrias do Setor de Petróleo e Gás Natural.

\section{Matérias e Métodos}

Os materiais usados neste trabalho foram o metal de adição AWS ER NiCrMo-3 (UNS N06625), com diâmetro de 1,2 mm e chapas de aço ASTM A516 Gr. 60 como metal de base. A composição química de ambos os materiais está apresentada na Tabela 1. Foi utilizado um eletrodo não consumível de tungstênio dopado com tório. 0 diâmetro do eletrodo de tungstênio foi de $4 \mathrm{~mm}$. 0 gás de proteção utilizado foi argônio puro com vazão de 15,0 l/min.. Para a realização das soldagens foi utilizado uma fonte eletrônica multiprocesso, um cabeçote para alimentação automática de arame frio, uma tocha TIG acoplada com um sistema de ajuste da posição do arame (Figura 1), e um robô industrial (Figura 2). 
Tabela 1. Composição química do metal de base e do metal de adição segundo o fabricante.

\begin{tabular}{|c|c|c|c|c|c|c|c|c|}
\hline \multirow{2}{*}{$\begin{array}{c}\text { Item } \\
\text { ASTM A516 Gr60 }\end{array}$} & \multicolumn{8}{|c|}{ Composição, peso \% } \\
\hline & $\mathbf{N i}$ & C & $\mathrm{Cr}$ & Mo & $\mathbf{F e}$ & Al & Mn & Si \\
\hline & 0,01 & 0,15 & 0,02 & 0,01 & Bal. & 0,02 & 0,95 & 0,2 \\
\hline & \multicolumn{8}{|c|}{ Composição, peso \% } \\
\hline \multirow[t]{4}{*}{ AWS ERNiCrMo-3 } & $\mathrm{Ni}$ & C & $\mathrm{Cr}$ & Mo & $\mathbf{F e}$ & $\mathrm{Cu}$ & Al & Ti \\
\hline & 61 & 0,05 & 21,5 & 9 & 2,5 & - & 0,2 & 0,2 \\
\hline & Nb & Mn & $\mathbf{S i}$ & & & & & \\
\hline & 3,6 & 0,2 & 0,2 & & & & & \\
\hline
\end{tabular}

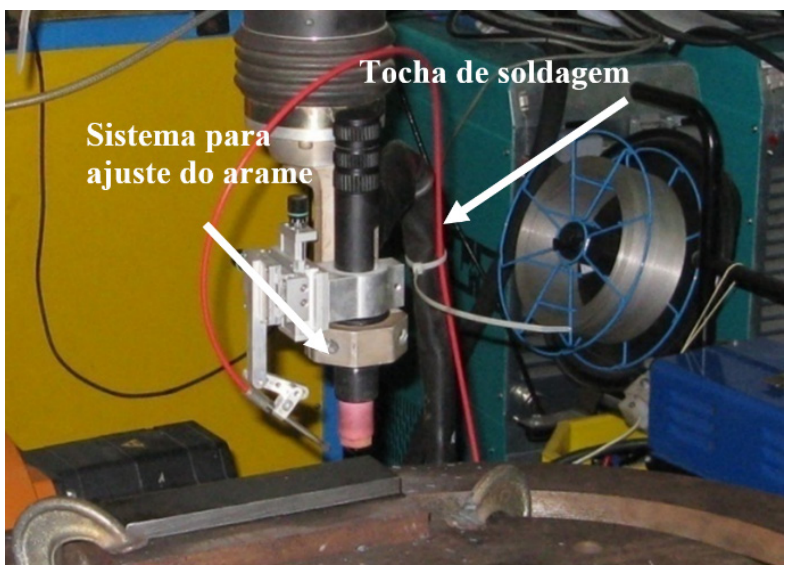

Figura 1. Tocha de soldagem com sistema para ajuste do arame.

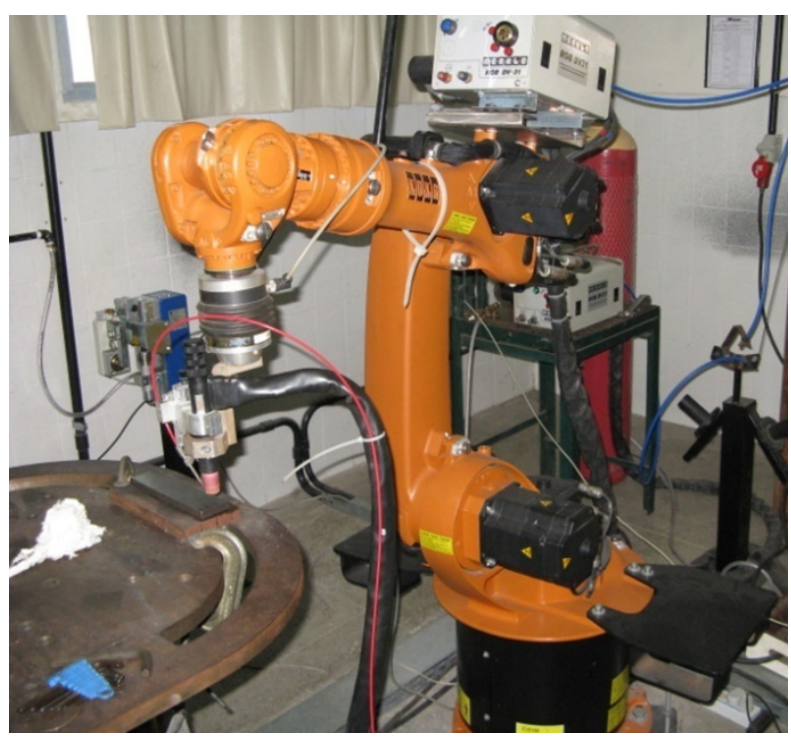

Figura 2. Robô industrial.

As soldagens de simples deposição foram realizadas na posição plana sobre chapas de aço carbono ASTM A516 Gr. 60 nas dimensões de $200 \times 50 \times 12,5 \mathrm{~mm}$. Para avaliar o efeito do uso de tecimento sobre as características geométricas e diluição do cordão de solda, inclusive para diferentes níveis de energia de soldagem, foram adotadas três diferentes níveis de energia para a deposição de cordões simples, os quais foram executados sem e com o uso do tecimento, conforme a Tabela 2. No caso do tecimento foi usada o tipo triangular, o qual descreve uma trajetória conforme apresentado na Figura 3. Foi ajustada uma amplitude de $4,0 \mathrm{~mm}$, um passo de 2,0 mm, e uma 
Avaliação do Uso do Tecimento sobre o Nível de Diluição e Geometria do Cordão de Solda na Soldagem TIG com Alimentação Automática de Arame Frio

Tabela 2. Parâmetros para soldagem utilizando o metal de adição AWS ERNiCrMo-3.

\begin{tabular}{cclcc}
\hline Ensaio & Condição & \multicolumn{1}{c}{ Tecimento } & $\begin{array}{c}\text { Corrente de solda- } \\
\text { gem (A) }\end{array}$ & Energia (kJ/cm) \\
1 & CC1 & Sem Tecimento & 223 & 9,6 \\
2 & CC1T & Tecimento Triangular & 223 & 9,6 \\
3 & CC2 & Sem Tecimento & 285 & 12,3 \\
4 & CC2T & Tecimento Triangular & 285 & 12,3 \\
5 & CC3 & Sem Tecimento & 343 & 14,8 \\
6 & CC3T & Tecimento Triangular & 343 & 14,8 \\
\hline
\end{tabular}

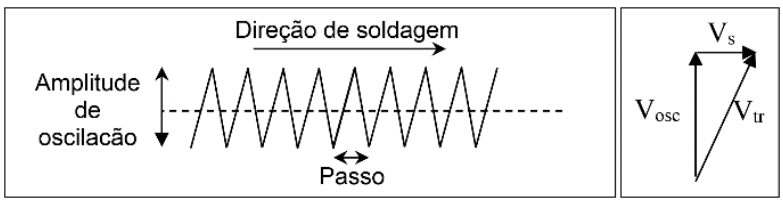

Figura 3. Desenho esquemático do movimento descrito no tecimento triangular.

frequência de 2,08 Hz. Devido a mudança de trajetória, a velocidade é decomposta em velocidade de oscilação e velocidade de deslocamento transversal para permitir o movimento transversal do arco. A velocidade de soldagem (Vs) foi ajustada em $25 \mathrm{~cm} / \mathrm{min}$. De acordo com os parâmetros de tecimento foi calculada a velocidade de oscilação $\left(V_{\text {osc }}\right)$, com base na Equação 1 [21], cujo valor foi de $100 \mathrm{~cm} / \mathrm{min}$. Neste caso, o tempo de parada lateral foi zero. Aplicando-se o conceito do teorema de Pitágoras é possível correlacionar as três componentes de velocidade. A velocidade de deslocamento transversal $\left(V_{t r}\right)$ foi calculada em 103,1 cm/min (Equação 2).

Durante a realização das soldagens foi utilizado um sistema de aquisição dos sinais de tensão e de corrente em 12 bits e a uma taxa de $9,6 \mathrm{kHz}$. O tempo de aquisição de cada ensaio foi de 5 segundos. $O$ início da aquisição dos dados de cada ensaio ocorreu no momento em que o arco estivesse mais estabilizado, aproximadamente 10 segundos após a abertura do arco.

Para realização deste estudo outras importantes variáveis do processo foram mantidas constante: a velocidade de alimentação de $6 \mathrm{~m} / \mathrm{min}$; o ângulo de alimentação do arame em $50^{\circ}$ em relação ao eixo do eletrodo de W (Figura 4); a Distância Ponta do Arame a Peça (DPAP) em 3 mm; e a Distância Ponta do Eletrodo a Peça (DPEP) em $6 \mathrm{~mm}$.

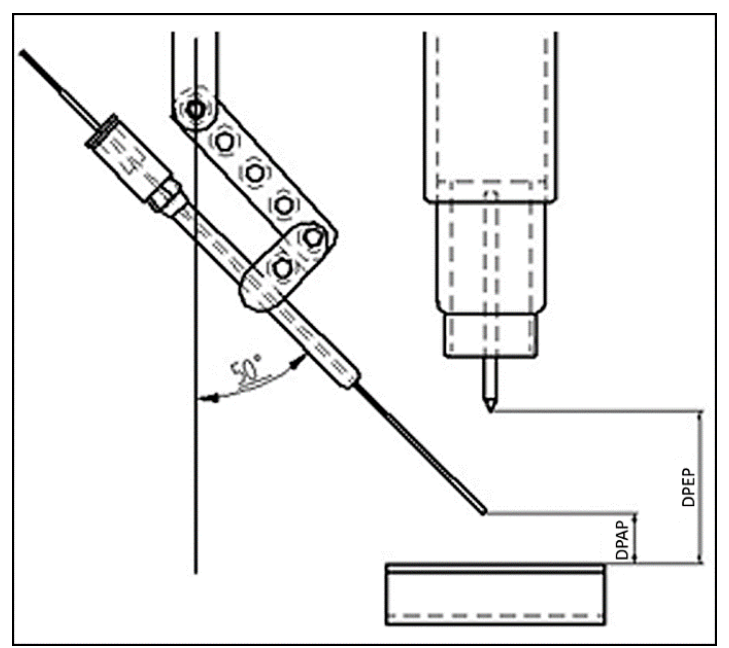

Figura 4. Desenho esquemático do ângulo de alimentação. 


$$
\begin{aligned}
& V_{O s c}=\frac{2 f A}{1-2 f t_{p l}} \\
& \left(V_{T r}\right)^{2}=\left(V_{O s c}\right)^{2}+\left(V_{S}\right)^{2}
\end{aligned}
$$

Após as soldagens, os corpos de prova foram submetidos à preparação metalográfica para obtenção das características geométricas (reforço, largura e penetração) e dos valores de diluição para as condições avaliadas. O comprimento total do cordão de solda foi de $180 \mathrm{~mm}$. Para a análise metalográfica foram retiradas três seções do corpo de prova, de $10 \mathrm{~mm}$ cada, no início, meio e fim, descartando $15 \mathrm{~mm}$ em cada borda do cordão.

A preparação das amostras consistiu em um lixamento usando uma lixadeira rotativa com lixas de granulação de $80,100,220,320,400,600$ e 1200 mesh. A etapa de lixamento foi seguida pelo uso de uma politriz para polimento com pasta de diamante de $3 \mu \mathrm{m}$, seguido de um ataque químico com nital $2 \%$ e tempo de ataque de 20 s, para revelação do perfil geométrico do cordão de solda.

Para análise da geometria e diluição foi utilizado um programa de análise de imagens comercial. Este programa consiste em realizar a medida da largura, reforço e penetração, a partir da macrografia da amostra com uma escala em milímetros servindo como referência. A diluição foi medida através da relação entre as áreas adicionada e diluída, de acordo com a Equação 3. A Figura 5 mostra as características geométrica medidas. De forma a verificar a influência dos fatores de controle sobre as variáveis de resposta foi realizada uma avaliação estatística empregando análise de variância (ANOVA).

$$
D(\%)=\frac{A_{M B}}{A_{M B}+A_{M A}} \times 100
$$

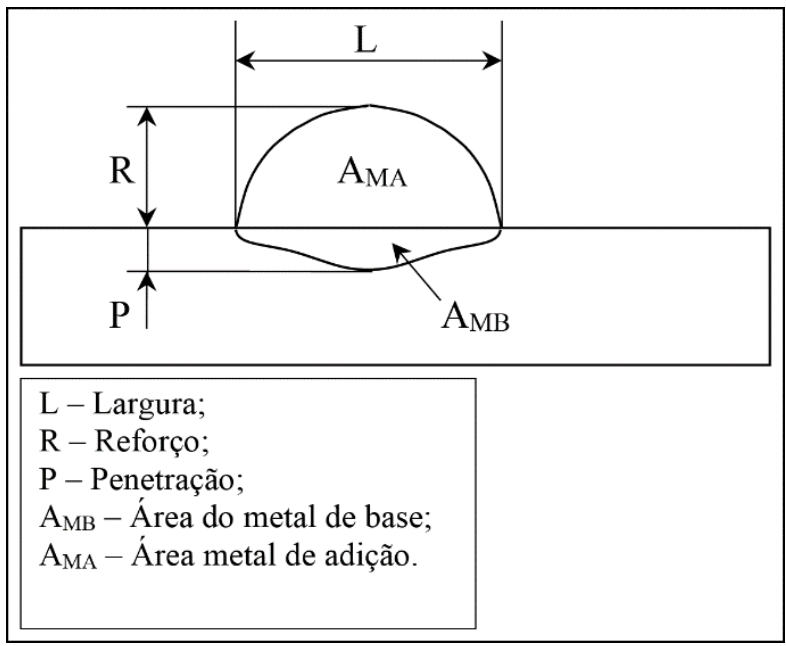

Figura 5. Geometria típica de um cordão de solda simples e as principais medidas geométricas.

\section{Resultados e Discussão}

Os resultados do efeito do tecimento sobre as características geométricas e diluição de cordões de solda de ligas de níquel do tipo AWS ER NiCrMo-3 são apresentados a seguir. A Tabela 3 mostra os resultados relacionados à geometria do cordão de solda e ao nível de diluição. Por esta tabela pode-se observar que na condição de baixa energia o nível de diluição variou de 5,37\% sem o uso do tecimento, para 1,52\% com uso do tecimento, comportamento este que se repetiu para os outros dois níveis de energia. Observa-se também que o uso do tecimento, além de reduzir significativamente os valores de diluição, também alterou de forma significativa o reforço, a largura e a penetração em relação as condições sem tecimento. 
Avaliação do Uso do Tecimento sobre o Nível de Diluição e Geometria do Cordão de Solda na Soldagem TIG com Alimentação Automática de Arame Frio

Tabela 3. Resultado das soldagens do fatorial completo com corrente contínua.

\begin{tabular}{|c|c|c|c|c|c|c|c|c|c|c|}
\hline Ensaio & Cond. & Tec. & Pos. & $\begin{array}{c}E \\
(\mathrm{~kJ} / \mathrm{cm})\end{array}$ & D (\%) & $\begin{array}{c}\text { Média. D } \\
(\%)\end{array}$ & Ref. (mm) & $\begin{array}{l}\text { Larg. } \\
(\mathrm{mm})\end{array}$ & Pen. (mm) & Ref. /Larg. \\
\hline 1 & $\mathrm{CC} 1$ & 1 & Inicio & 1 & 4,94 & & 4,68 & 3,92 & 0,55 & 1,19 \\
\hline 1 & $\mathrm{CC} 1$ & 1 & Meio & 1 & 4,44 & $5,37 \pm 1,20$ & 4,52 & 4,48 & 0,43 & 1,01 \\
\hline 1 & $\mathrm{CC} 1$ & 1 & Fim & 1 & 6,73 & & 4,4 & 3,78 & 0,71 & 1,16 \\
\hline 2 & $\mathrm{CC} 1$ & 2 & Inicio & 1 & 1,71 & & 3,91 & 8,97 & 0,13 & 0,44 \\
\hline 2 & $\mathrm{CC} 1$ & 2 & Meio & 1 & 1,67 & $1,52 \pm 0,29$ & 3,87 & 8 & 0,12 & 0,48 \\
\hline 2 & $\mathrm{CC} 1$ & 2 & Fim & 1 & 1,18 & & 3,89 & 8,15 & 0,08 & 0,48 \\
\hline 3 & $\mathrm{CC} 2$ & 1 & Inicio & 2 & 14,75 & & 4,33 & 6,23 & 1,30 & 0,70 \\
\hline 3 & $\mathrm{CC} 2$ & 1 & Meio & 2 & 13,25 & $13,13 \pm 1,68$ & 4,13 & 6,78 & 1,08 & 0,61 \\
\hline 3 & $\mathrm{CC} 2$ & 1 & Fim & 2 & 11,4 & & 4,31 & 6,12 & 1,10 & 0,71 \\
\hline 4 & $\mathrm{CC} 2$ & 2 & Inicio & 2 & 8,54 & & 3,48 & 8,70 & 0,35 & 0,40 \\
\hline 4 & $\mathrm{CC} 2$ & 2 & Meio & 2 & 8,71 & $8,28 \pm 0,60$ & 3,74 & 7,89 & 0,50 & 0,47 \\
\hline 4 & $\mathrm{CC} 2$ & 2 & Fim & 2 & 7,58 & & 3,50 & 8,12 & 0,40 & 0,43 \\
\hline 5 & $\mathrm{CC} 3$ & 1 & Inicio & 3 & 29,4 & & 3,46 & 10,35 & 1,86 & 0,33 \\
\hline 5 & $\mathrm{CC} 3$ & 1 & Meio & 3 & 28,45 & $28,79 \pm 0,53$ & 3,09 & 10,02 & 1,71 & 0,31 \\
\hline 5 & $\mathrm{CC} 3$ & 1 & Fim & 3 & 28,53 & & 3,14 & 10,71 & 1,64 & 0,29 \\
\hline 6 & $\mathrm{CC} 3$ & 2 & Inicio & 3 & 20,16 & & 2,98 & 11,89 & 0,76 & 0,25 \\
\hline 6 & $\mathrm{CC} 3$ & 2 & Meio & 3 & 19,81 & $18,87 \pm 1,94$ & 2,81 & 11,81 & 0,67 & 0,24 \\
\hline 6 & $\mathrm{CC} 3$ & 2 & Fim & 3 & 16,64 & & 3,01 & 11,74 & 0,65 & 0,26 \\
\hline
\end{tabular}

Onde: Pos. - posição de onde foi retirado o corpo de prova; E - Energia; D - diluição; Med. D. - média da diluição; Ref.- reforço; Larg.- largura; Pen- penetração.

A Figura 6 mostra as seções transversais dos cordões soldados com e sem tecimento. Pode-se observar que as amostras na condição sem tecimento apresentam uma penetração mais acentuada na porção central do fundo da poça, denominado de efeito taça. Também é possível constatar visualmente que o uso do tecimento reduziu consideravelmente a diluição. Isso ocorre pelo fato do movimento da poça distribuir melhor o calor ao longo de uma maior área, reduzindo o aquecimento localizado. Além disso, o uso do tecimento auxilia no espalhamento do metal líquido, o que proporciona uma importante alteração na geometria do cordão, tornando-o menos convexo, como pode ser observado na Figura 5. Com isso, torna mais fácil produzir a sobreposição de cordões para a formação de uma camada de revestimento. Igualmente importante é a redução na tendência de formação de defeitos entre passes na soldagem multipasse com objetivo de produção de camadas de revestimentos.

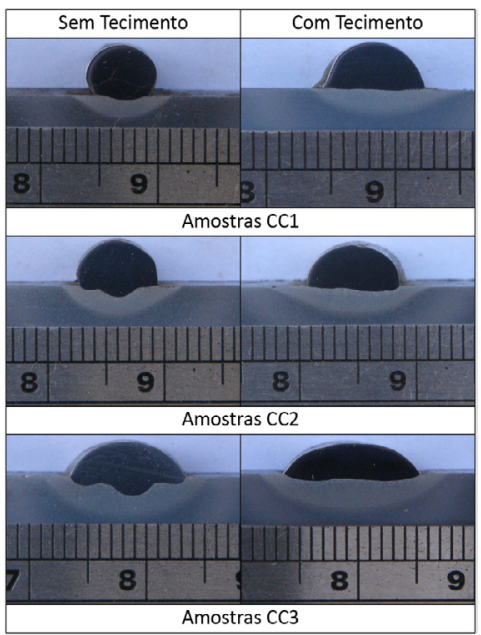

Figura 6. Seção transversal das amostras soldadas na condição CC1, CC2, CC3 e CC4 com DPEP de 6 mm extraídas do centro da chapa. 
O que foi constatado através da Figura 6 será validado graficamente a seguir através de análise estatística de variância, na qual se verifica a influência dos fatores de controle sobre as variáveis de resposta. Os níveis de significância " $\alpha$ " menores que 0,05 indicam que os fatores de controle tiveram influência estatisticamente significativa sobre as variáveis de resposta, com confiabilidade de $95 \%$.

\subsection{Influência do tecimento sobre a geometria do cordão de solda}

Os valores de " $\alpha$ " calculados a partir da análise de variância considerando a influência do tecimento sobre as variáveis de resposta indicaram um efeito estatisticamente significativo para todas as características geométricas avaliadas, inclusive sobre a diluição. A Figura 7 mostra o efeito do tipo de tecimento sobre o reforço do cordão de solda. Analisando a Figura 7, foi possível verificar uma redução no reforço do cordão de solda quando utiliza o tecimento, confirmando o que já era esperado, pois o uso do tecimento proporciona uma melhor distribuição de material sobre a peça, o que implica na redução do reforço e, consequentemente, no aumento da largura do cordão de solda, cujo comportamento também foi analisado com base na Figura 8.

Considerando inicialmente os valores médios para o reforço, observa-se uma queda no valor desta característica geométrica de 4,0 mm para aproximadamente $3,3 \mathrm{~mm}$. Esta redução embora aparente ser pequena, representa uma queda de $21 \%$ na altura do cordão, e na prática implica em redução de material nobre depositado, impactando diretamente sobre os custos de operação. Importante destacar que mesmo com a queda a espessura da camada ainda está dentro do especificado por norma para a soldagem de revestimentos com ligas resistentes à corrosão, que determina uma espessura mínima de 3,0 mm [14].

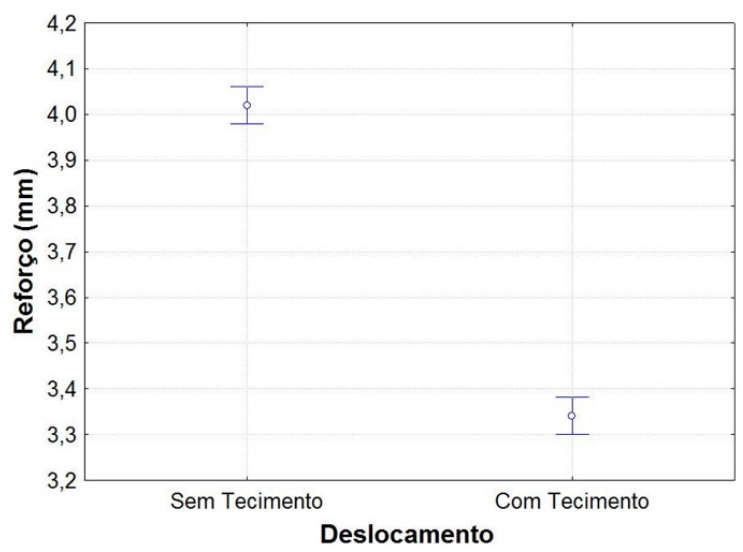

Figura 7. Influência do Tecimento sobre o Reforço do Cordão de Solda.

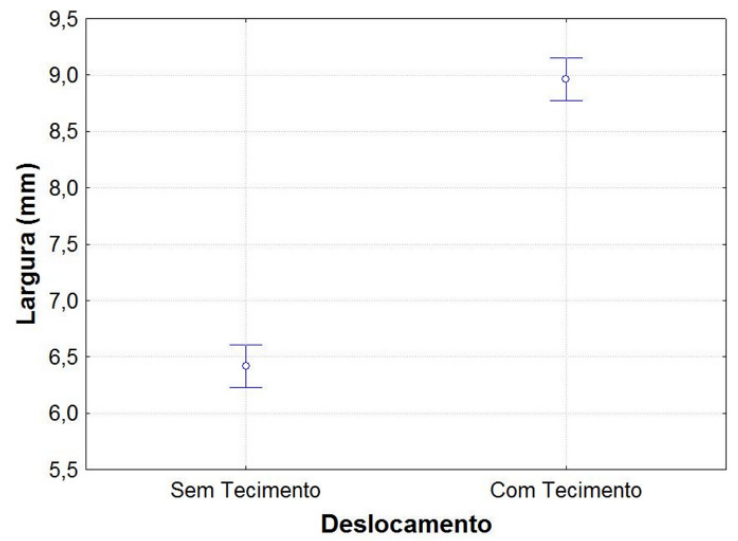

Figura 8. Influência do Tecimento sobre a Largura do Cordão de Solda. 
Avaliação do Uso do Tecimento sobre o Nível de Diluição e Geometria do Cordão de Solda na Soldagem TIG com Alimentação Automática de Arame Frio

Outra importante característica a ser avaliada é a largura do cordão de solda. Conforme destacado anteriormente, esta variável resposta apresentou um aumento estatisticamente significativo, cujo comportamento pode ser visualizado com base na Figura 8. É possível observar que a largura aumenta de aproximadamente $6,4 \mathrm{~mm}$ para a condição sem tecimento para quase $9,0 \mathrm{~mm}$ com o uso do mesmo. Esta variação representa um aumento de $40 \%$ na largura do cordão de solda, contribuindo significativamente para o aumento da área revestida. Por este motivo, o uso do tecimento proporciona uma maior área de cobertura por kg de material depositado, se comparado à técnica de deposição sem o movimento transversal. Isto implica em ganhos expressivos em termos de produtividade e redução dos custos por reduzir o tempo de operação [22].

A Figura 9 mostra a influência do tecimento sobre a penetração do cordão de solda. É possível observar uma redução significativa da penetração de $0,82 \mathrm{~mm}$ para $0,27 \mathrm{~mm}$ com o uso do tecimento. Este resultado corresponde a uma queda de $67 \%$ referente à esta variável resposta. Tal comportamento confirma o que já foi encontrado por Miranda [22]. O tecimento além de proporcionar uma melhor distribuição de material sobre a peça, conforme comentado anteriormente, proporciona também uma melhor distribuição de calor sobre a mesma, acarretando com isso nas reduções na penetração e na diluição.

Em termos de diluição, observou-se um comportamento de queda com o uso do tecimento seguindo a mesma linha observada para a penetração. Com base na Figura 10 é possível observar que na média, com o uso do tecimento a diluição caiu de aproximadamente $11 \%$ para apenas $6 \%$. Este resultado mostra uma queda de quase $45 \%$ no valor médio da diluição para a aplicação do tecimento. Considerando especificamente a condição de soldagem CC1T que corresponde a menor energia de soldagem aplicada com o uso do tecimento, foram obtidos níveis de diluição de apenas 1,52\%, como mostra a Figura 6.

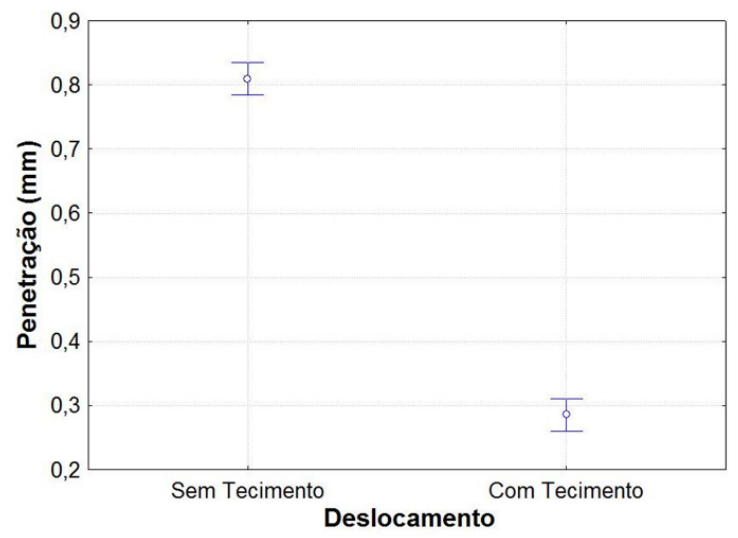

Figura 9. Influência do Tecimento sobre a Penetração.

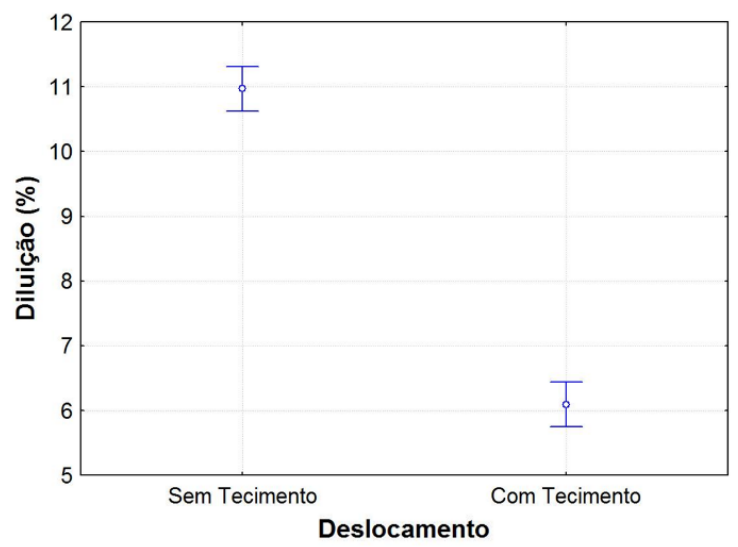

Figura 10. Influência do Tecimento sobre a Diluição. 
Este resultado é potencialmente benéfico, pois mostram a viabilidade de obtenção de cordões de solda com valores de diluição inferiores ou muito próximos do valor máximo requerido para a produção de revestimentos. De acordo com a determinação de normas internacionais para equipamentos para a indústria do petróleo [14], é recomendado um teor de Fe máximo na camada de revestimento de $5 \%$ em peso para revestimentos depositados com a liga 625. Em geral na indústria, o atendimento desta condição de teor de Fe é realizado somente com a deposição de duas ou mais camadas de revestimentos. Portanto, o presente estudo comprova que o uso do tecimento na soldagem de revestimento com ligas de níquel se mostrou fundamental para possibilitar a deposição de cordões de solda com diluição inferior a $2 \%$.

Embora não se tenha realizado análises de composição química para constatar o teor de Fe na zona fundida, vários trabalhos demonstram haver uma boa correlação entre a diluição obtida por análise química e análise geométrica, desde que realizada criteriosamente [11,12,23]. Vale ressaltar que outros parâmetros de soldagem como a distância da ponta do eletrodo à peça e a própria sobreposição lateral entre os cordões podem impactar positivamente na redução desta variável resposta.

Em outro trabalho estudando o efeito da distância da ponta do eletrodo à peça, Silva [23] verificaram que com o aumento deste fator de controle a diluição sofria uma redução que poderia chegar à $30 \%$, dependendo do valor ajustado. Outro importante resultado apontado no estudo de Silva [23] foi a identificação de um valor ótimo de $10 \mathrm{~mm}$ para a soldagem de revestimento com ligas à base de níquel buscando a redução da penetração e, principalmente, da diluição, o qual difere do valor utilizado no presente trabalho. Isto indica que a redução da diluição pode ser ainda maior se combinados os parâmetros de forma a maximizar os efeitos sobre a redução da fusão de metal de base que está diretamente vinculada à penetração e à diluição.

Contudo, vale ressaltar que na soldagem de revestimento exige-se por um lado um baixo nível de diluição, mas por outro um nível mínimo de diluição é necessário para se garantir a coalescência entre o revestimento e o substrato. Caso isso não ocorra, fatalmente ocorrerá o desprendimento do cordão de solda em relação ao substrato. Na Figura 11 é apresentado um exemplo de um cordão de solda de liga de níquel que se desprendeu do substrato, por falta de fusão. Neste caso, obviamente que os parâmetros não foram suficientes para transferir ao substrato o calor necessário para fusão. Porém, em relação ao nível mínimo de diluição é importante destacar que em outros resultados produzidos por Silva [23] verificou-se que condições em que se obteve nível de diluição tão baixo quanto $4 \%$ foram aprovadas em ensaio de cisalhamento, indicando que é possível produzir revestimentos com baixo nível de diluição preservando a alta aderência entre revestimento e substrato por meio das ligações químicas.

A Figura 12 mostra a influência do tecimento sobre a razão reforço/largura (R/L), onde pode-se observar uma redução substancial com o uso do tecimento. Este parâmetro é importante, pois indica o grau de convexidade do cordão de solda. Quanto menor a razão R/L menor será o grau de convexidade do cordão de solda, fato este que é benéfico para aplicação de revestimento, pois a sobreposição dos passes ocorre de forma mais homogênea, evitando assim a formação de possíveis vazios entre os passes, além de promover um melhor acabamento para a camada de revestimento.

Conforme apresentado na Figura 10, a redução nos valores de diluição é importante porque quanto menor este valor, mais próximo estará a composição química e as propriedades do revestimento das condições desejadas,

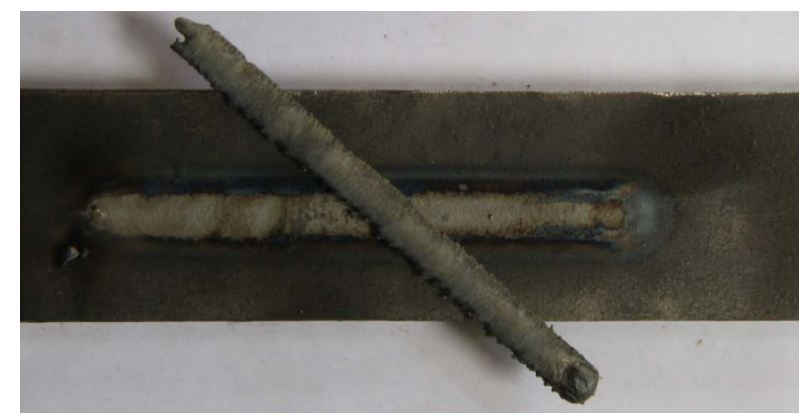

Figura 11. Cordão de solda de liga de níquel destacado do expontaneamente devido a falta de fusão e ausência de coalescencia com o substrato [22]. 
Avaliação do Uso do Tecimento sobre o Nível de Diluição e Geometria do Cordão de Solda na Soldagem TIG com Alimentação Automática de Arame Frio

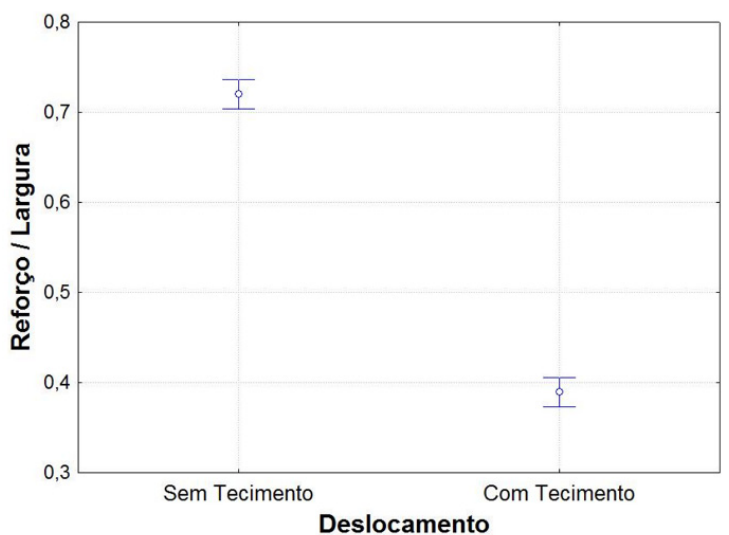

Figura 12. Influência do Tecimento sobre a razão Reforço/Largura.

ressaltando que altos níveis de diluição podem produzir efeitos indesejáveis ao metal de solda, especialmente em termos de resistência à corrosão [13,24,25]. Como foi verificado na Figura 12, a redução da razão reforço/largura (R/L) é bastante significativo com o uso do tecimento, este parâmetro é importante, pois indica o grau de convexidade do cordão de solda. Quando se tem uma razão R/L alta indica que o grau de convexidade é excessivo, dificultando com isso uma adequada sobreposição entre passes para soldagem de revestimentos com a possibilidade de geração de defeitos entre os passes [26].

Conforme os dados quantitativos apresentados, verifica-se um importante efeito do tecimento sobre todas as características geométricas avaliadas, inclusive sobre a diluição que constitui uma das principais características a ser otimizada na soldagem de revestimentos. Contudo, torna-se necessário destacar que os resultados quantitativos obtidos neste estudo se aplicam para a soldagem dissimilar com a liga AWS ER NiCrNo-3 (Inconel 625) depositada sobre aço baixo carbono, visto que alterações na composição química das ligas afetam propriedades como temperatura de fusão, molhabilidade e viscosidade, afetando as características de espalhamento da poça de fusão.

\section{4 . Conclusões}

Com base nos resultados apresentados neste trabalho sobre o efeito do Tecimento sobre a diluição e as características geométricas do cordão de solda depositado pelo processo TIG com alimentação de arame frio visando à soldagem de revestimentos, foi possível concluir que:

- Em soldagens robotizadas o uso do tecimento com controle rigoroso do movimento transversal do conjunto arco elétrico/poça de fusão se mostrou bastante favorável para a produção de soldas com baixos níveis de diluição em revestimentos com a liga AWS ER NiCrMo-3 (Inconel 625).

- O uso do tecimento reduz bastante a penetração, e como consequência também reduziu significativamente o nível de diluição, produzindo cordões com até $1,5 \%$ de diluição.

- O uso do tecimento também reduziu de forma considerável a razão reforço/largura (R/L), parâmetro importante, pois indica o grau de convexidade do cordão de solda e as características geométricas.

- A redução da convexidade dos cordões de solda devido o tecimento contribui decisivamente para minimizar o risco de formação de defeitos entre passes devido a sobreposição lateral de múltiplos passes para a formação de camadas de revestimentos.

\section{Agradecimentos}

Os autores deste trabalho agradecem ao Laboratório de Engenharia de Soldagem da UFC por toda infraestrutura para realização deste trabalho. À PETROBRAS pelo apoio e incentivo no desenvolvimento desta linha de pesquisa. Agradecem também a Fundação Cearense de Apoio ao Desenvolvimento Científico e Tecnológico - FUNCAP, ao CNPq, à CAPES e a FINEP pelo suporte financeiro. 


\section{Referências}

[1] Machado JPSE, Silva CC, Sobral-Santiago AVC, Sant'Ana HB, Farias JP. Effect of temperature on the level of corrosion caused by heavy petroleum on AISI 304 and AISI 444 stainless steel. Materials Research. 2006;9(2):137-142. http://dx.doi. org/10.1590/S1516-14392006000200005.

[2] Silva CC, Machado JPSE. Farias JP., Sobral AVC., Sant'ana HB., Rodrigues CEAL. Avaliação da corrosão do metal de solda de eletrodos revestidos AWS E309MoL-16 em meio contendo petróleo pesado. Tecnologica em Metalurgia, Materiais e Mineração. 2006;2(3):37-42. http://dx.doi.org/10.4322/tmm.00203008.

[3] Silva CC, Machado JPSE, Sobral-Santiago AVC, Sant'Ana HB, Farias JP. High-temperature hydrogen sulfide corrosion on the heat-affected zone of the AISI 444 stainless steel caused by Venezuelan heavy petroleum. Journal of Petroleum Science Engineering. 2007;59(3-4):219-225. http://dx.doi.org/10.1016/j. petrol.2007.04.003.

[4] Silva CC, Sant'Ana HB, Farias JP. Avaliação da resistência à corrosão causada por petróleo pesado em chapas soldadas utilizadas em "linings" de torres de destilação. Boletim Técnico da Petrobrás. 2008;51:09-35.

[5] Silva CC, Farias JP, Sant'Ana HB. Evaluation of AISI 316 L stainless steel welded plates in heavy petroleum environment. Materials \& Design. 2009;30(5):1581-1587. http://dx.doi.org/10.1016/j. matdes.2008.07.050.

[6] Silva CC, Sant'Ana HB, Miranda HC, Farias JP. Microstructure, hardness and petroleum corrosion evaluation of the AISI 316L/ AWS E309MoL-16 austenitic stainless steel dissimilar weld metal. Materials Characterization. 2009;60(4):346-352. http://dx.doi. org/10.1016/j.matchar.2008.09.017.

[7] Silva CC, Miranda HC, Sant'Ana HB, Farias JP. Austenitic and ferritic stainless steel dissimilar weld metal evaluation for the applications as-coating in the petroleum processing equipment. Materials \& Design. 2013;47:1-8. http://dx.doi.org/10.1016/j. matdes.2012.11.048.

[8] Eiselstein HL, Tillack DJ, editores. Loria superalloy 718,625 , and various derivates. Warrendale: TMS; 1991.

[9] Brooks CR. Heat Treatment, structure and properties of nonferrous alloys. Ohio: ASM, Metals Park; 1982. Nickel based alloys; p. 139-227.

[10] Silva CC, Afonso CRM, Ramirez AJ, Motta MF, Miranda HC, Farias JP. Aspectos metalúrgicos de revestimentos dissimilares com a superliga à base de níquel Inconel 625. Soldagem e Inspeção. 2012;17(3):251-263. http://dx.doi.org/10.1590/ S0104-92242012000300009.

[11] Dupont JN. Marder, AR. Dilution in single pass arc welds. Metallurgical and Material Transaction B. 1996;27(3):481-489.

[12] Banovic SW, Dupont JN, Marder AR. Dilution and microsegregation in dissimilar metal welds between super austenitic stainless steel and nickel base alloys. Science and Technology of Welding and Joining. 2002;7(6):374-383. http://dx.doi. org/10.1179/136217102225006804.

[13] Gittos MF, Gooch TG. Effect of iron dilution on corrosion resistance of $\mathrm{Ni}-\mathrm{Cr}-\mathrm{Mo}$ alloy cladding. British Corrosion Journal, UK. 1996;31(4):309-314. http://dx.doi.org/10.1179/ bcj.1996.31.4.309.

[14] International Organization for Standardization - ISO. ISO 10423: Petroleum and natural gas industries - driling and production equipment - wellhead and christmas tree equipment. 3 ed. Geneva: ISO; 2009.
[15] Balasubramanian V, Lakshminarayanan AK, Varahamoorthy $\mathrm{R}$, Babu S. Understanding the parameters controlling plasma transferred arc hardfacing using response surface methodology. Materials and Manufacturing Processes. 2008;23(7):674-682. http://dx.doi.org/10.1080/15560350802316744.

[16] Juang SC, Tarng YS. Process parameter selection for optimizing the weld pool geometry in the tungsten inert gas welding of stainless steel. Journal of Materials Processing Technology. 2002;122(1):3337. http://dx.doi.org/10.1016/S0924-0136(02)00021-3.

[17] Kannan T, Murugan N. Effect of flux cored arc welding process parameters on duplex stainless steel clad quality. Journal of Materials Processing Technology. 2006;176(1-3):230-239. http:// dx.doi.org/10.1016/j.jmatprotec.2006.03.157.

[18] Palani PK, Murugan N. Optimization of weld bead geometry for stainless steel claddings deposited by FCAW. Journal of Materials Processing Technology. 2007;190(1-3):291-299. http://dx.doi. org/10.1016/j.jmatprotec.2007.02.035.

[19] Tarng YS, Juang SC, Chang CH. The use of grey-based Taguchi methods to determine submerged arc welding process parameters in hardfacing. Journal of Materials Processing Technology. 2002;128(1-3):1-6. http://dx.doi.org/10.1016/ S0924-0136(01)01261-4.

[20] Chen JH, Chen PN, Lin CM, Chang CM, Chang YY, Wu W. Characterization of multi-element alloy claddings manufactured by the tungsten inert gas process. Surface and Coatings Technology. 2009;203(20-21):2983-2988. http://dx.doi.org/10.1016/j. surfcoat.2009.02.138.

[21] Fratari RQ, Schvartzman MMAM, Scotti A. Otimização dos parâmetros de tecimento para confecção de amanteigamento em chapas de aço ao carbono pelo processo TIG com arame AWS ER309L. Soldagem e Inspeção. 2010;15(3):209-217. http:// dx.doi.org/10.1590/S0104-92242010000300007.

[22] Miranda EC. Estudo exploratório de parâmetros de soldagem de revestimentos com ligas de níquel empregando o processo TIG com alimentação de arame frio [dissertação de mestrado]. Fortaleza: Departamento de Engenharia Metalúrgica e de Materiais, Universidade Federal do Ceará; 2009.

[23] Silva CC. Revestimentos de liga de níquel depositados pelo processo tig com alimentação de arame frio - aspectos operacionais e metalúrgicos [tese de doutorado]. Fortaleza: Departamento de Engenharia Metalúrgica e de Materiais, Universidade Federal do Ceará; 2010.

[24] Abioye TE, Mccartney DG, Clare AT. Laser cladding of Inconel 625 wire for corrosion protection. Journal of Materials Processing Technology. 2015;217:232-240. http://dx.doi.org/10.1016/j. jmatprotec.2014.10.024.

[25] Silva CC, Afonso CRM, Ramirez AJ, Motta MF, Miranda HC, Farias JP. Evaluation of the Corrosion Resistant Weld Cladding Deposited by the TIG Cold Wire Feed Process. Materials Science Forum. 2014;783-786:2822-2827. http://dx.doi.org/10.4028/ www.scientific.net/MSF.783-786.2822.

[26] Silva CC, Miranda EC, Motta MF, Miranda HC, Farias JP. Minimização de defeitos em revestimentos de superligas de níquel depositada pelo Processo TIG com alimentação de arame frio. Soldagem e Inspeção. 2014;19(4):323-332. http://dx.doi. org/10.1590/0104-9224/SI1904.05. 